\title{
Drug Reaction with Eosinophilia and Systemic Symptoms Syndrome Asso-
} ciated with Ethambutol use: A Case Report

Ajita Kapur ${ }^{1}$ and Harmeet Singh Rehan ${ }^{1, *}$

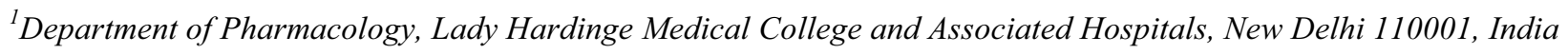

\begin{tabular}{l}
\hline A R T I C L E H I S T O R Y \\
\hline \\
Received: January 09, 2019 \\
Revised: February 25,2019 \\
Accepted: February 28, 2019 \\
DOI: \\
10.2174/1574886314666190307150757 \\
\end{tabular}

Abstract: Background: Among the first line Anti-Tubercular Drugs (ATDs), ethambutol has been
rarely associated to cause drug reaction with eosinophilia and systemic symptoms (DRESS) syndrome.

Keywords: Drug reaction with eosinophilia, systemic symptoms syndrome, ethambutol, adverse drug reaction, drug-induced, ATDs.

\section{INTRODUCTION}

Drug reaction with eosinophilia and systemic symptoms (DRESS) syndrome is a rare life-threatening idiosyncratic, multi-system adverse drug reaction characterized by diffuse infiltrated rash with facial edema, lymphadenopathy, eosinophilia, atypical lymphocytes, fever and involvement of visceral organs [1]. DRESS syndrome is associated with a mortality of $5 \%$ to $10 \%$ due to the involvement of internal organs, especially fulminant hepatitis [2]. Several drugs like lamotrigine, allopurinol, minocycline and sulfonamides have been implicated to cause DRESS [3]. DRESS syndrome due to antitubercular drugs (ATDs) is rare and often underdiagnosed. Identifying the incriminating ATD is often challenging as these are prescribed in combination. Management of ATD induced DRESS is difficult as withdrawal of drugs and initiation of corticosteroid lead to worsening of tuberculosis. We report a rare case of DRESS syndrome certainly associated to ethambutol use and successfully managed by ethambutol withdrawal and steroid therapy.

\section{CASE REPORT}

A 34-year-old woman presented to the out-patient department of dermatology with a history of fever associated with chills, rigors, itchy red raised lesions all over the body, swelling of the face, headache, dizziness and tinnitus for the last four days. The rashes appeared on the face and progressed to involve trunk, upper limb and lower limb within a day.

*Address correspondence to this author at the Department of Pharmacology, Lady Hardinge Medical College and Associated Hospitals, New Delhi 110001, India; Tel: +919811694040; E-mail: harmeetrehan@hotmail.com
Medication history revealed that she was on isoniazid $(\mathrm{H})$ $(5 \mathrm{mg} / \mathrm{kg})$, rifampicin (R) $(10 \mathrm{mg} / \mathrm{kg})$, pyrazinamide $(\mathrm{Z})$ $(25 \mathrm{mg} / \mathrm{kg})$, ethambutol (E) $(15 \mathrm{mg} / \mathrm{kg})$ and streptomycin $(\mathrm{S})$ $(15 \mathrm{mg} / \mathrm{kg})$ for the last 35 days for pulmonary tuberculosis (Fig. 1). She also revealed a past history of pulmonary tuberculosis 19 years back, which was successfully treated with HRZE for 2 months and HR for 4 months.

On clinical examination, the patient was febrile $\left(101^{\circ} \mathrm{F}\right)$ and submandibular, axillary and inguinal lymph nodes were enlarged. Multiple maculopapular erythematous lesions were present over the face, trunk, upper and lower extremities. She had facial edema, dry lips and aphthous ulcers.

Hematological investigations revealed that the total leukocyte count (TLC) was raised $(15000 / \mu 1)$, differential leukocyte count [neutrophils (46\%), eosinophils (30\%), lymphocytes $(18 \%)$ and monocytes $(6 \%)]$ was suggestive of eosinophilia [absolute eosinophil counts (AEC), 4500/ $\mu 1$ ]. Peripheral smear showed mild anisocytosis of red blood cells. Liver function test showed raised aspartate aminotransferase (AST) $(112 \mathrm{IU} / \mathrm{L}$, normal range $<31 \mathrm{IU} / \mathrm{L})$, low total serum proteins $(6 \mathrm{~g} / \mathrm{dl}$, normal range $=6.4-8.3 \mathrm{~g} / \mathrm{dl})$ and decreased serum albumin $(3.2 \mathrm{~g} / \mathrm{dl}$, normal range $=3.5-5.2 \mathrm{~g} / \mathrm{dl})$. Serology for dengue and malaria was negative. Blood and urine culture was sterile. Fasting and postprandial blood sugar levels, lipid profile, kidney function tests and serum electrolytes were within normal range. Skin biopsy showed mild focal spongiosis with mild perivascular lymphocytes and neutrophils infiltration. Chest radiography showed hyper-inflated lung fields with bilateral hilar lymphadenopathy. Noncontrast computed tomography (CT) head was normal. A diagnosis of DRESS was made.

Pulmonary medicine consultant advised the withdrawal of anti-tubercular therapy immediately and recommen-ded a 


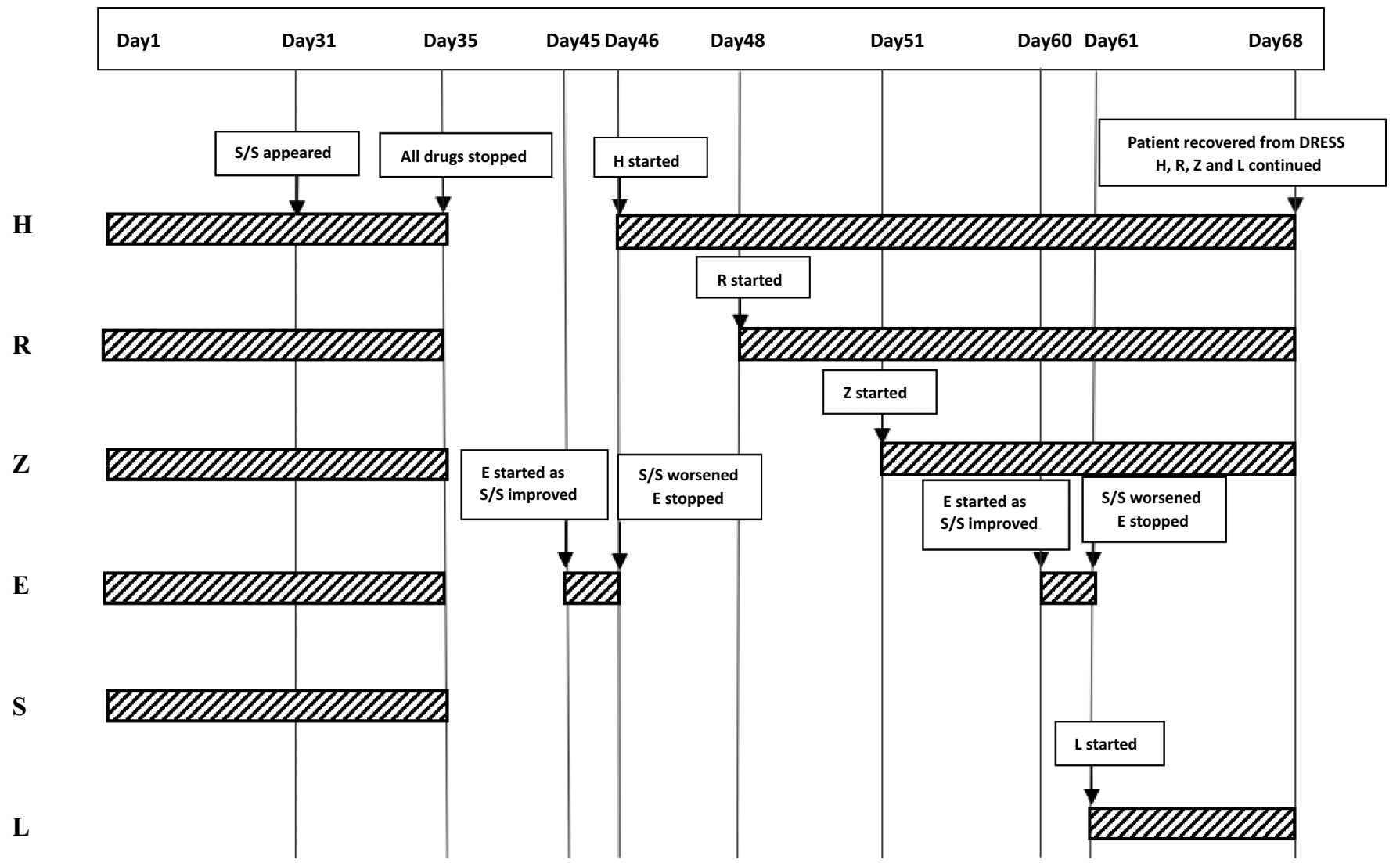

Fig. (1). Medication history of the patient (S/S: signs and symptoms, DRESS: Drug reaction with eosinophilia and systemic symptoms, H: isoniazid, R: rifampicin, Z: pyrazinamide, E: ethambutol, S: streptomycin, L: levofloxacin).

follow up after the resolution of skin lesions. The dermatologist advised the administration of hydrocortisone $100 \mathrm{mg}$ intravenously 8 hourly for one day, tablet prednisolone $40 \mathrm{mg}$ once daily per orally for three days followed by tapering of the dose, tablet paracetamol $500 \mathrm{mg}$ per orally one as needed, tablet cetirizine $10 \mathrm{mg}$ per orally one daily, cream fusidic acid $2 \%$ for topical application, cream fluticasone $0.05 \%$ for topical application and calamine lotion for local application on the skin lesion. The patient became afebrile $\left(98.6^{\circ} \mathrm{F}\right) \mathrm{im}-$ mediately whereas skin lesions and facial edema started resolving after two days of treatment. After ten days, ethambutol (800mg) was re-introduced as it is least known to implicate DRESS. But within 12 hours, her periorbital edema and pruritus worsened. Her TLC $(16900 / \mu 1)$ and AEC $(4800 / \mu 1)$ raised further. Ethambutol was stopped immediately and tablet isoniazid $(50 \mathrm{mg}$ ) was started (day 1$)$. The dose of isoniazid was increased to $300 \mathrm{mg}$ on the next day (day 2). On day 3, capsule rifampicin $150 \mathrm{mg}$ was added with a gradual increase in dose to $300 \mathrm{mg}$ and $450 \mathrm{mg}$ on day 4 and 5 respectively. On day 6 , tablet pyrazinamide $500 \mathrm{mg}$ once daily was introduced with a subsequent increase to twice a day. She tolerated $H, R$ and $Z$ well for the next ten days and her skin lesions and facial edema improved. The lower dose of ethambutol $(100 \mathrm{mg})$ was introduced again. Within a day, skin lesions worsened with the appearance of new urticarial lesions. Immediately ethambutol was replaced with a second line anti-tubercular drug, levofloxacin $(750 \mathrm{mg}$ per orally once daily). Patient's symptoms improved and cutaneous lesions recovered completely after a week.

\section{DISCUSSION}

The etiology of DRESS is not fully understood but is related to delayed immunological reactions to drugs and certain non-drug factors like genetic polymorphisms, human leucocyte antigen and herpesvirus reactivation. Shiohara et al. suggested that the polymorphisms in genes encoding drug metabolizing enzymes, such as cytochrome P450 enzyme and $\mathrm{N}$-acetyltransferase, may participate in the pathophysiology of DRESS [4]. Due to non-specific clinical features of DRESS mimicking infectious, neoplastic or rheumatologic diseases it is difficult to diagnose DRESS [5]. Recently, several diagnostic criteria for DRESS have been recommended $[6,7]$. The diagnosis of definite case of DRESS (total score of 7) was made as per European Registry of Severe Cutaneous Adverse Reactions to Drugs and Collection of Biological Samples (RegiSCAR) group [6] criteria in our patient as she had enlarged lymph nodes of size $>1 \mathrm{~cm}$ at four sites (score of 1), fever of $\geq 38.5^{\circ} \mathrm{C}$ (score of 0 ), rash with facial edema and purpura (score of 1), involving whole body surface area (score of 1), eosinophilia (AEC of 4500/ $\mu 1,30 \%$ ) (score of 2), disease duration of 38 days (score of 0 ), raised AST (112 $\mathrm{IU} / \mathrm{L})$, decreased serum proteins and albumin $(6 \mathrm{~g} / \mathrm{dl}$ and 3.2 $\mathrm{g} / \mathrm{dl}$ ) and hyper-inflated lung fields (score of 2), mild focal spongiosis with mild perivascular lymphocytes and neutrophils infiltration in skin biopsy (score of 0 ) and negative blood and urine cultures (score of 0 ). 
This patient developed initial symptoms of DRESS i.e. rash with facial edema and fever after five weeks of starting the first line anti-tubercular medications. The temporal relation of the appearance of signs and symptoms of DRESS has been reported to be within three weeks to three months of initiation of an offending drug [4]. Skin lesions in this case resolved on discontinuation of ethambutol along with other anti-tubercular drugs and on the administration of systemic and topical steroids. Palmero et al. reported the role of systemic corticosteroid in the resolution of ATD induced DRESS without the worsening of tuberculosis [5].

An initial re-administration of ethambutol $(800 \mathrm{mg})$ was done after 10 days of stopping ATDs. Ethambutol was preferred as the first drug for re-challenge as it is least associated to cause DRESS [8]. Similar to a previously reported case of ethambutol-induced DRESS [9], in this patient, worsening of skin lesions was seen within 12 hours of ethambutol readministration. Ethambutol was stopped again immediately following which lesions improved. After 15 days of resolution of lesions, second re-challenge with a lower dose of ethambutol (100mg) was done. This led to worsening of resolving lesions with the appearance of new rashes. Ethambutol (100mg) was withdrawn, rifampicin, isoniazid, and pyrazinamide were restarted and levofloxacin, a second line anti-tubercular drug was added. Husain et al. also recommended management of patients with ATD induced DRESS by withdrawing the offending agents and introducing second-line anti-tubercular drugs [10].

There is a high likelihood for the recurrence of DRESS following the re-administration of offending medication [5]. Though Siripassorn et al. attempted successful desensitization to anti-tuberculosis drugs in a case of DRESS [11]. In such situations, it is ethical to conduct a patch test to identify the suspected offending agent. This patient took six-month anti-tubercular therapy nineteen years back which was free from any such untoward event. There should be alertness for bizarre adverse drug reactions, which can rarely occur on subsequent exposures after such a long time.

\section{CONCLUSION}

Based on the above available facts, there was a reasonable temporal relation between the event and the administration of offending drug i.e. ethambutol and no other illness or concomitant drug could explain the signs and symptoms of DRESS. Further, in this case, de-challenge and re-challenge with ethambutol were positive. As per the World Health Organization-Uppsala Monitoring Centre (WHO-UMC) causality assessment scale [12], the association between ethambutol and DRESS was certain. The association was definite according to Naranjo scale [13].

Patients who develop DRESS to one agent may have an increased risk of DRESS to same and other unrelated drugs [5, 14]. Clinicians should be watchful to avoid re-exposure of patients with a history of DRESS to the suspected offending drug.

\section{ETHICS APPROVAL AND CONSENT TO PARTICI- PATE}

Not applicable.

\section{HUMAN AND ANIMAL RIGHTS}

Not applicable.

\section{CONSENT FOR PUBLICATION}

Not applicable.

\section{STANDARD FOR REPORTING}

The CARE guidelines and methodologies were followed in this study.

\section{FUNDING}

None.

\section{CONFLICT OF INTEREST}

The authors declare no conflict of interest, financial or otherwise.

\section{ACKNOWLEDGEMENTS}

Declared none.

\section{REFERENCES}

[1] Kardaun SH, Sekula P, Valeyrie-Allanore L, et al. Drug reaction with eosinophilia and systemic symptoms (DRESS): An original multisystem adverse drug reaction. Results from the prospective RegiSCAR study. Br J Dermatol 2013; 169(5): 1071-80.

[2] Cacoub P, Musette P, Descamps V, et al. The DRESS syndrome: A literature review. Am J Med 2011; 124(7): 588-97.

[3] Duong TA, Valeyrie-Allanore L, Wolkenstein P, Chosidow O. Severe cutaneous adverse reactions to drugs. Lancet 2017; 390(10106): 1996-2011.

[4] Shiohara T, Kano Y. Drug reaction with eosinophilia and systemic symptoms (DRESS): Incidence, pathogenesis and management. Expert Opin Drug Saf 2017; 16(2): 139-47.

[5] Palmero D, Castagnino J, Musella RM, Mosca C, González Montaner $\mathrm{P}$, de Casado GC. Difficult clinical management of anti-tuberculosis DRESS syndrome. Int J Tuberc Lung Dis 2013; 17(1): 76-8.

[6] Kardaun SH, Sidoroff A, Valeyrie-Allanore L, et al. Variability in the clinical pattern of cutaneous side-effects of drugs with systemic symptoms: Does a DRESS syndrome really exist? Br J Dermatol 2007; 156 : 609-11.

[7] Shiohara T, Inaoka M, Kano Y. Drug-induced hypersensitivity syndrome (DIHS): A reaction induced by a complex interplay among herpesviruses and antiviral and antidrug immune responses. Allergol Int 2006; 55: 1-8.

[8] Allouchery M, Logerot S, Cottin J, et al. Antituberculosis drugassociated DRESS: A case series. J Allergy ClinImmunol Pract 2018; 6(4): 1373-80.

[9] Gest N, Ingen-Housz-Oro S, Gener G, et al. Drug reaction with eosinophilia and systemic symptoms (DRESS) syndrome due to ethambutol. Med Mal Infect 2018; 48(4): 302-5.

[10] Husain Z, Reddy BY, Schwartz RA. DRESS syndrome: Part II Management and therapeutics. J Am Acad Dermatol 2013; 68(5): 709.

[11] Siripassorn K, Ruxrungtham K, Manosuthi W. Successful drug desensitization in patients with delayed-type allergic reactions to anti-tuberculosis drugs. Int J Infect Dis. 2018; 68: 61-8.

[12] The use of WHO-UCM system for standardized case causality assessment. Available from http://www.who.int/medicines/ areas/quality_safety/safety_efficacy/WHOcausality_assessment.pdf (accessed on 2018 Sept 4)

[13] Naranjo CA, Busto U, Sellers EM, et al. A method for estimating the probability of adverse drug reactions. Clin Pharmacol Ther 1981; 30(2): 239-45.

[14] Picard D, Vellar M, Janela B, et al. Recurrence of drug-induced reactions in DRESS patients. J Eur Acad Dermatol Venereol 2015; 29: 801-4. 